\title{
Solución salina hipertónica y dosis altas de diurético, ¿qué sabemos y cómo podemos usarlos en congestión persistente?
}

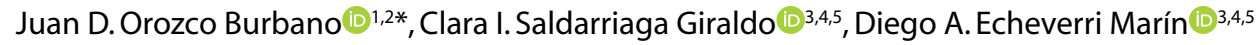

Recibido: 17 de octubre de 2021.

Aceptado: 20 de noviembre de 2021

Filiación de los autores

Departamento de Medicina

Interna, Universidad del Cauca.

Cauca, Colombia.

Hospital Universitario San José

Popayán, Colombia,

Departamento de Cardiología,

Universidad Pontificia Bolivariana. Medellín, Colombia.

Clínica CardioVID. Medellín

Colombia.

Universidad de Antioquia. Mede-

llín, Colombia.

*Correspondencia

*Juan D. Orozco Burbano

Calle 28 CN \# 6 c 72 . Popayán, Cauca

- Colombia.

Correo

jdorozco@unicauca.edu.co

Financiamiento

Los autores declaran no tener finan-

ciamiento alguno para la presente

revisión.

Conflictos de interés

Los autores declaran no tener

conflictos de interés.

Citar como

Orozco Burbano JD, Saldarriag

Giraldo Cl, Echeverri Marín DA.

Solución salina hipertónica y dosis

altas de diurético, ¿qué sabemos

y cómo podemos usarlos en

congestión persistente? Arch Peru

Cardiol Cir Cardiovasc. 2021;2(4):247

253. doi: 10.47487/apcyccv.v2i4.159.

\section{RESUMEN}

La presencia de una descompensación en falla cardiaca continúa siendo una condición con altas tasas de hospitalización, impacto en el sistema de salud y calidad de vida de quienes la padecen. El eje principal del tratamiento en estos casos son los diuréticos; sin embargo, en ocasiones se puede presentar resistencia a este grupo farmacológico, lo que genera un inadecuado balance negativo de líquidos y persistencia de la congestión con desenlaces clínicos negativos. La solución salina hipertónica junto con dosis altas de diurético surge como una opción terapéutica para este grupo de pacientes, con probables beneficios fisiológicos, clínicos e impacto en tasas de hospitalización y reingreso por descompensación de falla cardiaca. A continuación, se realiza una revisión de los aspectos más relevantes y beneficios de esta combinación.

Palabras clave: Insuficiencia cardíaca; Solución salina hipertónica; Furosemida (fuente: DeCS BIREME).

\section{ABSTRACT}

\section{Hypertonic saline solution and high dose of diuretic, ¿what do we know and how can we use them in persistent congestion?}

The presence of decompensated heart failure continues to be a condition with high rates of hospitalization, impact on the health system, and quality of life for those who suffer it. The mainstay of treatment in these cases are diuretics. However, the resistance to this pharmacological group may occasionally occur, generating an inadequate negative fluid balance and persistence of congestion with negative clinical outcomes. Hypertonic saline solution with high doses of diuretic emerges as a therapeutic option for this group of patients with probable physiological, and clinical benefits on hospitalization and re-admission rates due to heart failure decompensation. A review of the most relevant aspects and benefits of this combination is discussed in this article.

Keywords: Heart failure; Saline solution, hypertonic; Furosemide (source: MeSH NLM). 


\section{Introducción}

La prevalencia de falla cardiaca continúa en incremento debido al aumento en la expectativa de vida y factores de riesgo cardiovascular ${ }^{(1)}$. Esta condición puede presentar estados de descompensación, debido a la presencia de un evento agudo (de novo) o a un empeoramiento de signos y síntomas de una falla preexistente asociados con una marcada sobrecarga de volumen ${ }^{(2)}$. La falla cardiaca descompensada (FCD) representa, en países desarrollados, más de 1 millón de hospitalizaciones al año, con altos costos para el sistema de salud ${ }^{(3)}$, mientras que en países de mediano y bajos ingresos, los datos acerca de la prevalencia son limitados y se obtienen de registros hospitalarios ${ }^{(4)}$

La presencia de congestión en pacientes con FCD se ha asociado con diversos mecanismos poco conocidos ${ }^{(5)}$ que conllevan a la persistencia de sobrecarga de volumen intravascular y redistribución en diversos tejidos. Algunos de los mecanismos asociados pueden ser inherentes al paciente (no adherencia a terapias tanto farmacológicas como no farmacológicas) o alteraciones fisiopatológicas propias de la falla cardiaca (hiperaldosteronismo secundario y lesión renal aguda) ${ }^{(6,7)}$. El pilar fundamental para favorecer la descongestión en FCD es el manejo con diuréticos de asa como la furosemida; sin embargo, por los mecanismos previamente mencionados, se pueden generar alteraciones en la farmacodinamia y farmacocinética de los diuréticos, presentándose una respuesta inadecuada o resistencia a este grupo farmacológico, prolongando la estancia hospitalaria y el incremento en la mortalidad de estos pacientes ${ }^{(6-9)}$.

En este contexto, la solución salina hipertónica (SSH) junto con diuréticos de asa, han surgido como estrategia en casos de resistencia diurética, asociándose a optimización en la descongestión en FCD, desenlaces clínicos favorables, sin mayor impacto en la función renal ${ }^{(8-10)}$. Presentamos una revisión acerca del mecanismo de acción, beneficios documentados hasta el momento e indicaciones de la solución salina hipertónica en FCD.

\section{Metodología}

Las bases de datos que se revisaron fueron PubMed, Google Scholar y SciELO, estableciendo un límite de tiempo de 20 años, sin restricción idiomática (desde el 01/09/2001 hasta el 01/09/2021). Las palabras clave utilizadas fueron: heart failure, saline solution hypertonic y furosemide, las cuales fueron combinadas con el término boleano "AND". La búsqueda arrojó un total de 160 artículos, de ellos se tomaron 37 , los cuales incluían a las palabras clave en su título principal o en su resumen. Se incluyó un ensayo clínico aleatorizado fundamental para la revisión por fuera del tiempo estipulado realizado por Paterna S. et al. en el año 2000, con lo cual se consideraron 38 artículos. Se excluyeron los artículos que no tuvieran disponible el texto completo o no tuvieran relación con el objetivo de esta revisión, también los estudios duplicados, los únicamente descriptivos o reportes de casos.

La resistencia a diuréticos, un problema por vencer con solución salina hipertónica (SSH)

Los diuréticos de asa son la piedra angular en el tratamiento de la FCD; habitualmente, una dosis de $\mathbf{4 0} \mathrm{mg}$ de furosemida debe generar una diuresis entre 3 a $4 L^{(11)}$. No obstante, en ocasiones no se logra una óptima descongestión o balances negativos, a pesar de dosis adecuadas y crecientes de diurético. Este efecto se ha denominado resistencia a diurético y puede catalogarse como una pérdida de respuesta o reducción en la respuesta a este grupo farmacológico ${ }^{(7)}$.

Entre un 20 a $50 \%$ de los pacientes hospitalizados por falla cardiaca descompensada pueden presentar resistencia diurética, lo que se ha asociado independientemente con un aumento en las tasas de mortalidad a 180 días (HR: 1,42; IC del 95\%: 1,11-1,81, $p=0,005$ ) y hospitalización por falla cardiaca a 60 días (HR: 1,57; IC del 95\%: 1,24-2,01, $p<0,001)^{(12)}$. Este fenómeno de resistencia se encuentra con menos frecuencia en pacientes con falla cardiaca leve y función renal conservada ${ }^{(7)}$.

No está claro el mecanismo de producción de resistencia diurética en $\mathrm{FCD}^{(12)}$; sin embargo, se postula que existe una probable adaptación funcional del túbulo contorneado distal, que ocurre posterior al inicio de furosemida, con una disminución de la respuesta a dosis posteriores, fenómeno denominado «frenado» ${ }^{(13,14)}$. Por otra parte, en FCD se requieren mayores dosis de diurético con el fin de lograr una respuesta natriurética máxima, situación que se exacerba cuando existe compromiso renal, y se crea una relativa resistencia a los diuréticos (Figura 1) ${ }^{(14-17)}$. Respecto a la presencia de acidosis tubular e hipoalbuminemia, estas dos condiciones se han asociado con una secreción alterada de diuréticos en los túbulos proximales; sin embargo, estas pueden ser irrelevantes en los mecanismos de resistencia en FCD ${ }^{(18)}$.

\section{Beneficios asociados a la solución salina hipertónica con} altas dosis de diurético

La SSH en combinación con altas dosis de furosemida, se ha considerado como terapia para restaurar la eficacia farmacológica de los diuréticos ${ }^{(19,20)}$. El mecanismo de acción principal, por el cual la SSH ejerce un efecto es a través de un mecanismo osmótico que favorece un gradiente positivo desde el espacio intersticial 


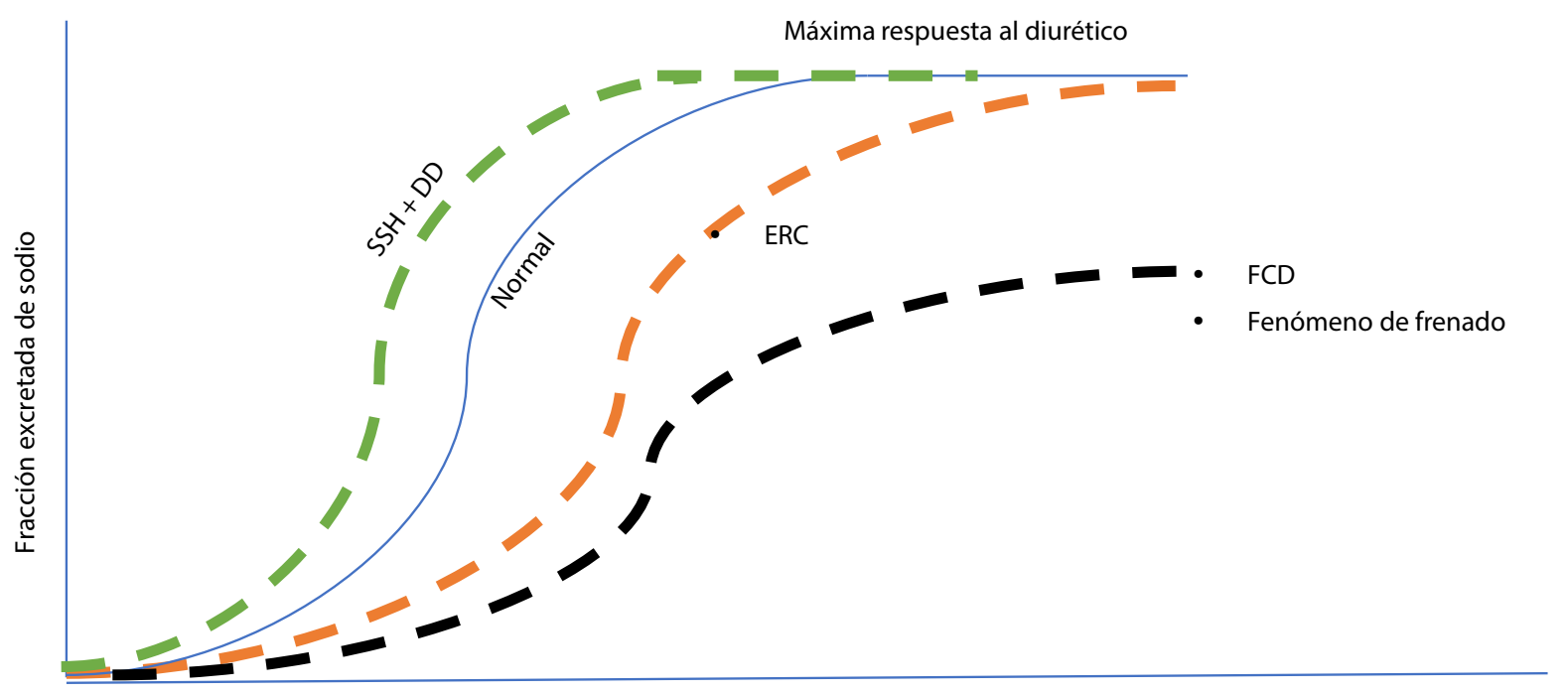

Concentración de diurético (dosis furosemida)

SSH: solución salina hipertónica. DD: dosis alta de diurético. ERC: enfermedad renal crónica. FCD: falla cardiaca descompensada. Adaptado de Ref. 14-17.

Figura 1. Curva dosis/efecto respuesta a furosemida. Efecto de la solución salina hipertónica más dosis alta de diurético.

al intravascular, con el subsecuente arrastre de agua libre ${ }^{(8)}$. Esto genera un aumento hasta en $30 \%$ del volumen intraplasmático, favoreciendo la perfusión miocárdica y renal, situación que optimiza la precarga y disminuye la poscarga, además, produce una disminución de la presión en cuña de la arteria pulmonar ${ }^{(19,21)}$.

A nivel cardíaco, se ha reportado beneficios del uso de la SSH caracterizado por un probable efecto inotrópico directo y una reducción del edema miocárdico con incremento de captación de calcio por parte de los miocitos y una restauración del potencial transmembrana ${ }^{(22)}$. Además, se ha demostrado que la SSH produce una mejoría en marcadores cardiacos, generando una disminución de los niveles de troponina, NTpro BNP (NT-proB-type Natriuretic Peptide, por sus siglas en inglés) y péptidos natriuréticos atriales ${ }^{(13,23,24)}$.

En el ámbito renal, la administración de la SSH se ha asociado con una mejoría en la función tubular con disminución de los marcadores de daño renal temprano y un probable efecto que evita el deterioro en la función renal durante la terapia diurética intensiva, asociado al incremento de la perfusión renal durante la FCD ${ }^{(20,25,26)}$. El aumento de sodio a nivel tubular distal, contrarresta la retención de sodio posterior al túbulo proximal, evitando el fenómeno de «frenado» ${ }^{(27)}$. Otro mecanismo asociado de la SSH es el aumento en los niveles de factor de necrosis tumoral alfa (TNF-a) a nivel tubular, lo cual evita la excesiva reabsorción de sodio en la rama ascendente gruesa del asa de Henle, mitigando la sobrecarga de volumen ${ }^{(28)}$.
Por otro lado, se ha encontrado que la SSH incrementa la presencia de furosemida intraluminal y la excreción de sodio en el túbulo contorneado distal, generando desviación de la curva dosis/efecto hacia la izquierda ${ }^{(16,17)}$, como se demuestra en la Figura 1. Además, el uso de SSH se ha asociado con una disminución en la reducción de terapias invasivas como la ultrafiltración o hemodiálisis en pacientes con falla cardiaca avanzada ${ }^{(29)}$. Otro punto importante del uso de la SSH en FCD, ha sido la probable asociación con la reducción en el tiempo de hospitalización, con un promedio de 3,5 días, disminución en reingresos $(R R, 0,50 ; 95 \% \mathrm{Cl}, 0,33-0,76 ; \mathrm{p}<0,05)$ y mortalidad asociada a $F C D(R R, 0,46 ; 95 \% \mathrm{Cl}, 0,36-0,59){ }^{(17,30-32)}$. La Figura 2 resume los efectos potenciales de la SSH ${ }^{(19)}$.

Protocolo en FCD con SSH y dosis altas de diuréticos, ¿cómo usarla y en quién?

Existen diversos protocolos para el uso de SSH más altas dosis de diurético (Tabla 1) ${ }^{(21,22,27,33,34)}$. Esta combinación se debe usar, idealmente, en pacientes con FCD con fracción de eyección reducida en quienes, a pesar del uso de altas dosis de furosemida (250 a 500 mg/día) y diuréticos (tiazídicos y espironolactona) no logren una óptima descongestión ${ }^{(35)}$. El protocolo de Paterna et al. ${ }^{(33)}$, actualmente se aplica en la mayoría de los pacientes candidatos, el cual consiste en la administración de $150 \mathrm{~mL}$ de SSH dos veces al día y dosis altas de furosemida (500-1000 mg, dos veces al día), tanto la SSH como la furosemida se deben 


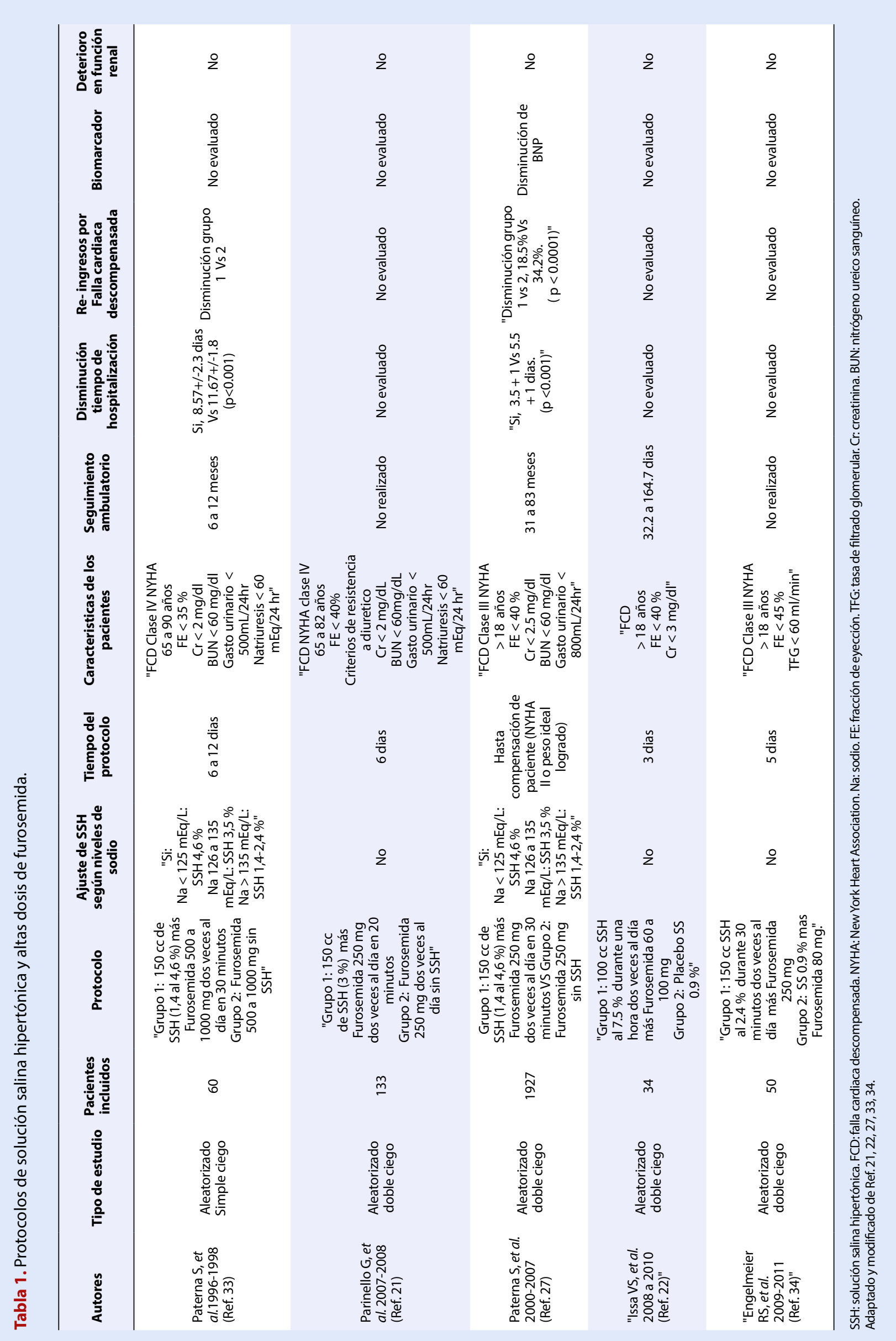



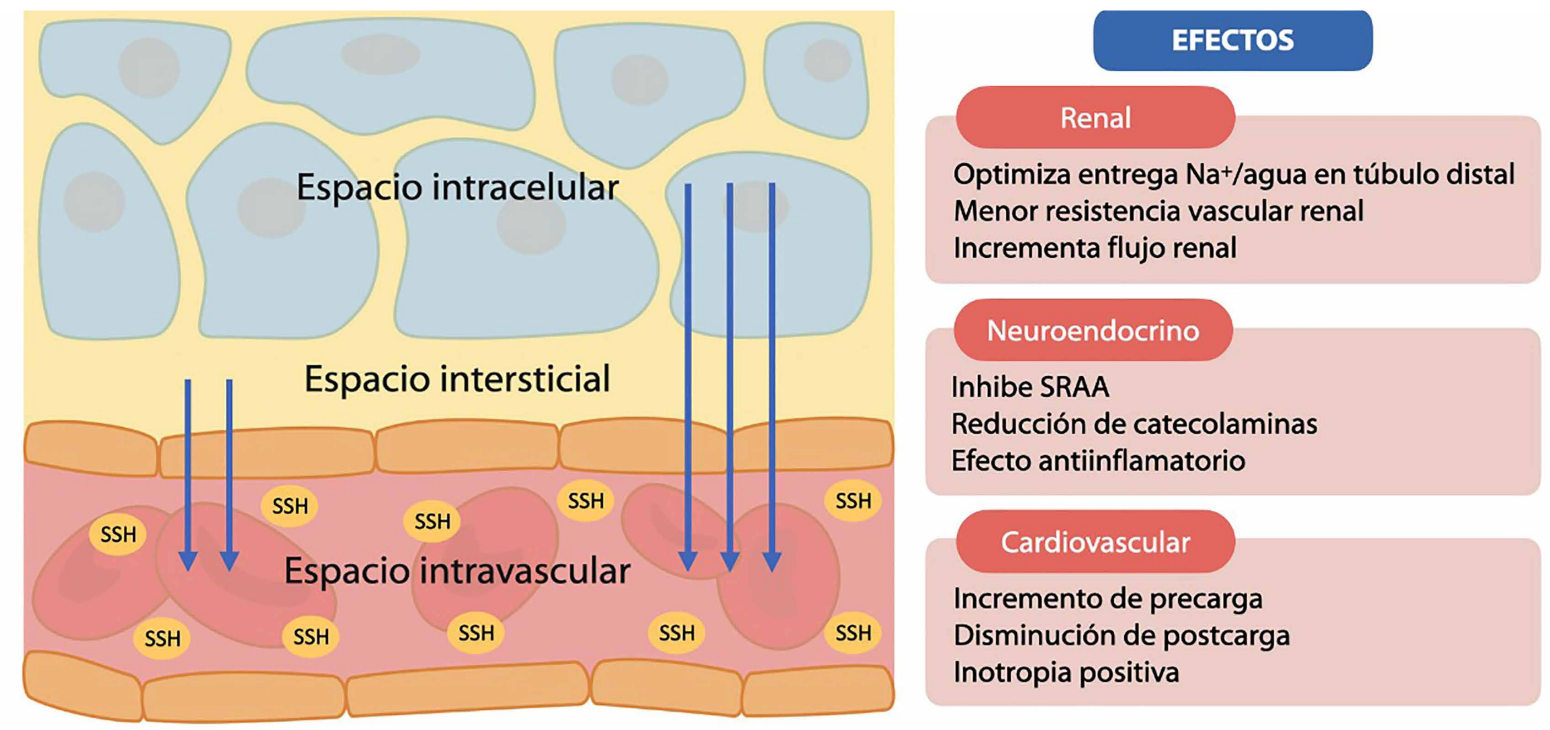

SRAA: sistema renina angiotensina aldosterona. $\mathrm{Na+}$ : sodio. Fuente: elaboración propia. Adaptado de Ref. 19.

Figura 2. Esquema del mecanismo de acción y potenciales efectos de la solución salina hipertónica.

pasar de manera conjunta durante $30 \mathrm{~min}$, protocolo que debe ser llevado durante seis días ${ }^{(32,33,36)}$.

Para la administración de esta combinación, se debe tener en cuenta algunas condiciones del paciente como los valores de sodio, pues la hiponatremia puede ocurrir entre un 19 a $25 \%$ de pacientes con FCD ${ }^{(37)}$. Por lo tanto, dependiendo del sodio sérico se modificará la concentración de SSH (Tabla 2). Otra condición para tener en cuenta es la función renal del paciente, aunque se conoce que la mayoría de los pacientes con FCD y compromiso renal pueden generar mayor resistencia a los diuréticos ${ }^{(7)}$ los niveles de creatinina (Cr) no influyen sobre la dosis de furosemida y de $\mathrm{SSH}$; no obstante, se debe limitar su uso cuando se tengan niveles de $\mathrm{Cr}>2,5 \mathrm{mg} / \mathrm{dL}^{(29,36,38)}$.

\section{Conclusiones}

La SSH junto con dosis altas de diuréticos, surge como opción terapéutica en pacientes con falla cardiaca descompensada, principalmente en el subgrupo de pacientes con resistencia a

Tabla 2. Protocolos de solución salina hipertónica según concentración de sodio.

\begin{tabular}{|c|c|c|}
\hline $\begin{array}{l}\text { Niveles de sodio del } \\
\text { paciente }\end{array}$ & Concentración de SSH & Modo de preparación de SSH (opciones) \\
\hline$<125 \mathrm{mEq} / \mathrm{L}$ & $4,6 \%$ & $\begin{array}{l}\text { 1. Diluir } 250 \mathrm{cc} \text { de } \mathrm{SS} 0,9 \% \text { más } 18 \text { ampollas de cloruro de sodio ( } 20 \\
\mathrm{meq} / 10 \mathrm{~mL} \text { ) } \\
\text { 2. Diluir } 300 \mathrm{cc} \text { de agua destilada más } 20 \text { ampollas de cloruro de } \\
\text { sodio }(20 \mathrm{meq} / 10 \mathrm{~mL} \text { ) }\end{array}$ \\
\hline$>126 \mathrm{a} \leq 135 \mathrm{mEq} / \mathrm{L}$ & 2,4 a $3,5 \%$ & $\begin{array}{l}\text { 1. Diluir } 400 \mathrm{cc} \text { de } \mathrm{SS} 0,9 \% \text { más } 10 \text { ampollas de cloruro de sodio ( } 20 \\
\text { meq } / 10 \mathrm{~mL} \text { ) } \\
\text { 2. Diluir } 370 \mathrm{cc} \text { de agua destilada más } 13 \text { ampollas de cloruro de } \\
\text { sodio }(20 \mathrm{meq} / 10 \mathrm{~mL} \text { ) }\end{array}$ \\
\hline$\geq 136 \mathrm{mEq} / \mathrm{L}$ & $1,4 \%$ & $\begin{array}{l}\text { 1. Diluir } 450 \mathrm{cc} \text { de } \mathrm{SS} 0,9 \% \text { más } 3 \text { ampollas de cloruro de sodio ( } 20 \\
\mathrm{meq} / 10 \mathrm{~mL} \text { ) } \\
\text { 2. Diluir } 440 \mathrm{cc} \text { de agua destilada más } 6 \text { ampollas de cloruro de sodio } \\
(20 \mathrm{meq} / 10 \mathrm{~mL})\end{array}$ \\
\hline
\end{tabular}

SSH: solución salina hipertónica; SS: solución salina. Adaptado y modificado de Ref. 36. 
diuréticos. Este protocolo puede ser aplicado fácilmente en la mayoría de centros hospitalarios; hasta el momento es el manejo farmacológico sin mayores efectos adversos y con probables beneficios clínicos junto con la reducción de hospitalizaciones y reingresos por falla cardiaca descompensada.

\section{Contribuciones de los autores}

JOB: diseño del estudio, extracción de los datos, revisión y redacción del manuscrito. CSG, DEM: revisión, redacción del manuscrito y aprobación de la versión final del manuscrito.

\section{Referencias bibliográficas}

1. Joseph SM, Cedars AM, Ewald GA, Geltman EM, Mann DL. Acute decompensated heart failure: contemporary medical management. Texas Heart Inst J. 2009;36(6):510-20. Erratum in: Tex Heart Inst J. 2010;37(1):135.

2. Mangini S, Pires PV, Braga FG, Bacal F. Decompensated heart failure. Einstein (Sao Paulo). 2013 Jul-Sep;11(3):383-91. doi: 10.1590/s167945082013000300022.

3. Arrigo M, Jessup M, Mullens W, Reza N, Shah AM, Sliwa K, Mebazaa A. Acute heart failure. Nat Rev Dis Primers. 2020 Mar 5;6(1):16. doi: 10.1038/s41572-020-0151-7.

4. Writing Group Members, Mozaffarian D, Benjamin EJ, Go AS, Arnett DK, Blaha MJ, et al. Executive Summary: Heart Disease and Stroke Statistics--2016 Update: A Report from the American Heart Association. Circulation. 2016 Jan 26;133(4):447-54. doi: 10.1161/ CIR.0000000000000366.

5. Njoroge JN, Teerlink JR. Pathophysiology and Therapeutic Approaches to Acute Decompensated Heart Failure. Circ Res. 2021 May 14;128(10):1468-1486. doi: 10.1161/CIRCRESAHA.121.318186.

6. Scott MC, Winters ME. Congestive Heart Failure. Emerg Med Clin North Am. 2015 Aug;33(3):553-62. doi: 10.1016/j.emc.2015.04.006.

7. Shah N, Madanieh R, Alkan M, Dogar MU, Kosmas CE, Vittorio TJ. A perspective on diuretic resistance in chronic congestive heart failure. Ther Adv Cardiovasc Dis. 2017 Oct;11(10):271-278. doi: $10.1177 / 1753944717718717$.

8. Bowman BN, Nawarskas JJ, Anderson JR. Treating Diuretic Resistance: An Overview. Cardiol Rev. 2016 Sep-Oct;24(5):256-60. doi: 10.1097/ CRD.0000000000000116.

9. Griffin M, Soufer A, Goljo E, Colna M, Rao VS, Jeon S, et al. Real World Use of Hypertonic Saline in Refractory Acute Decompensated Heart Failure: A U.S. Center's Experience. JACC Heart Fail. 2020 Mar;8(3):199208. doi: 10.1016/j.jchf.2019.10.012.

10. De Vecchis R, Esposito C, Ariano C, Cantatrione S. Hypertonic saline plus i.v. furosemide improve renal safety profile and clinical outcomes in acute decompensated heart failure: A meta-analysis of the literature. Herz. 2015 May;40(3):423-35. doi: 10.1007/s00059013-4041-6.

11. Ter Maaten JM, Valente MA, Damman K, Hillege HL, Navis G, Voors AA. Diuretic response in acute heart failure-pathophysiology, evaluation, and therapy. Nat Rev Cardiol. 2015 Mar;12(3):184-92. doi: 10.1038/ nrcardio.2014.215.

12. Valente MA, Voors AA, Damman K, Van Veldhuisen DJ, Massie BM, $\mathrm{O}^{\prime}$ Connor $\mathrm{CM}$, et al. Diuretic response in acute heart failure: clinical characteristics and prognostic significance. Eur Heart J. 2014 May 14;35(19):1284-93. doi: 10.1093/eurheartj/ehu065.

13. Covic A, Copur S, Tapoi L, Afsar B, Ureche C, Siriopol D, et al. Efficiency of Hypertonic Saline in the Management of Decompensated Heart Failure: A Systematic Review and Meta-Analysis of Clinical Studies. Am J Cardiovasc Drugs. 2021 May;21(3):331-347. doi: 10.1007/ s40256-020-00453-7.

14. Tuttolomondo A, Pinto A, Parrinello G, Licata G. Intravenous highdose furosemide and hypertonic saline solutions for refractory heart failure and ascites. Semin Nephrol. 2011;31(6):513-522. doi: 10.1016/j. semnephrol.2011.09.006.

15. Casu G, Merella P. Diuretic Therapy in Heart Failure - Current Approaches. Eur Cardiol. 2015 Jul;10(1):42-47. doi: 10.15420/ ecr.2015.10.01.42.

16. Costanzo MR. Hypertonic Saline:The Genesis of the Exodus of Fluid in Heart Failure? JACC Heart Fail. 2020 Mar;8(3):209-211. doi: 10.1016/j. jchf.2019.11.010.

17. Paterna S, Di Gaudio F, La Rocca V, Balistreri F, Greco M, Torres D, et al. Hypertonic Saline in Conjunction with High-Dose Furosemide Improves Dose-Response Curves in Worsening Refractory Congestive Heart Failure. Adv Ther. 2015 Oct;32(10):971-82. doi: 10.1007/s12325-015-0254-9.

18. Gupta R, Testani J, Collins S. Diuretic Resistance in Heart Failure. Curr Heart Fail Rep. 2019 Apr;16(2):57-66. doi: 10.1007/s11897019-0424-1.

19. Liszkowski M, Nohria A. Rubbing salt into wounds: hypertonic saline to assist with volume removal in heart failure. Curr Heart Fail Rep. 2010 Sep;7(3):134-9. doi: 10.1007/s11897-010-0018-4.

20. Lafrenière $G$, Béliveau P, Bégin JY, Simonyan D, Côté $S$, Gaudreault V, et al. Effects of hypertonic saline solution on body weight and serum creatinine in patients with acute decompensated heart failure. World J Cardiol. 2017 Aug 26;9(8):685-692. doi: 10.4330/wjc. v9.i8.685.

21. Parrinello G, Paterna S, Di Pasquale P, Torres D, Mezzero M, Cardillo M, et al. Changes in estimating echocardiography pulmonary capillary wedge pressure after hypersaline plus furosemide versus furosemide alone in decompensated heart failure. J Card Fail. 2011 Apr;17(4):3319. doi: 10.1016/j.cardfail.2010.11.003.

22. Issa VS, Andrade L, Ayub-Ferreira SM, Bacal F, de Bragança AC, Guimarães GV, et al. Hypertonic saline solution for prevention of renal dysfunction in patients with decompensated heart failure. Int J Cardiol. 2013 Jul 15;167(1):34-40. doi: 10.1016/j.ijcard.2011.11.087.

23. Parrinello G, Di Pasquale P, Torres D, Cardillo M, Schimmenti C, Lupo $U$, et al. Troponin I release after intravenous treatment with high furosemide doses plus hypertonic saline solution in decompensated heart failure trial (Tra-HSS-Fur). Am Heart J. 2012 Sep;164(3):351-7. doi: 10.1016/j.ahj.2012.05.025.

24. Tuttolomondo A, Maida C, Casuccio A, Di Raimondo D, Fonte R, Vassallo V, et al. Effects of intravenous furosemide plus small-volume hypertonic saline solutions on markers of heart failure. ESC Heart Failure: 2021;411; 1-12. doi: https://doi.org/10.1002/ehf2.13511.

25. De Vecchis R, Ciccarelli A, Ariano C, Pucciarelli A, Cioppa C, Giasi $A$, et al. Renoprotektiver Effekt kleiner Volumina hypertoner Kochsalzlösung bei Patienten mit chronischer Herzinsuffizienz und Ödembildung: Ergebnisse einer Fall-Kontroll-Studie [Renoprotective effect of small volumes of hypertonic saline solution in chronic heart failure patients with marked fluid retention: results of a casecontrol study]. Herz. 2011 Feb;36(1):12-7. German. doi: 10.1007/ s00059-010-3394-3.

26. Issa VS, Bacal F, Mangini S, Dias Carneiro RM, Naves de Freitas Azevedo $\mathrm{CH}$, Chizzola PR, et al. Hypertonic saline solution for renal failure 
prevention in patients with decompensated heart failure. Arq Bras Cardiol. 2007 Oct;89(4):251-5. English, Portuguese. doi: 10.1590/ s0066-782×2007001600007.

27. Paterna S, Fasullo S, Parrinello G, Cannizzaro S, Basile I, Vitrano G, et al. Short-term effects of hypertonic saline solution in acute heart failure and long-term effects of a moderate sodium restriction in patients with compensated heart failure with New York Heart Association class III (Class C) (SMAC-HF Study). Am J Med Sci. 2011 Jul;342(1):2737. doi: 10.1097/MAJ.0b013e31820f10ad.

28. Gatzoflias $S$, Hao $S$, Ferreri NR. Induction of renal tumor necrosis factor- $a$ and other autacoids and the beneficial effects of hypertonic saline in acute decompensated heart failure. Am J Physiol Renal Physiol. 2021 Jun 1;320(6):F1159-F1164. doi: 10.1152/ ajprenal.00686.2020.

29. Gandhi S, Mosleh W, Myers RB. Hypertonic saline with furosemide for the treatment of acute congestive heart failure: a systematic review and meta-analysis. Int J Cardiol. 2014 May 1;173(2):139-45. doi: 10.1016/j.ijcard.2014.03.020.

30. Zepeda P, Rain C, Sepúlveda P. What are the effects of hypertonic saline plus furosemide in acute heart failure? Medwave. 2015 Aug 27;15 Suppl 2: e6233. English, Spanish. doi: 10.5867/ medwave.2015.6233.

31. Liu C, Peng Z, Gao X, Gajic O, Dong Y, Prokop LJ, et al. Simultaneous Use of Hypertonic Saline and IV Furosemide for Fluid Overload: A Systematic Review and Meta-Analysis. Crit Care Med. 2021 Nov 1:49(11):e1163-e1175. doi: 10.1097/CCM.0000000000005174.

32. Gómez Mesa JE, Saldarriaga C, Jurado AM, Mariño A, Rivera A, Herrera A, et al. Consenso colombiano de falla cardíaca avanzada: capítulo de Falla Cardíaca, Trasplante Cardíaco e Hipertensión Pulmonar de la Sociedad Colombiana de Cardiología y Cirugía Cardiovascular 2019; 26:3-24. doi: 10.1016/j.rccar.2019.06.001.

33. Paterna S, Di Pasquale P, Parrinello G, Amato P, Cardinale A, Follone G, et al. Effects of high-dose furosemide and small-volume hypertonic saline solution infusion in comparison with a high dose of furosemide as a bolus, in refractory congestive heart failure. Eur J Heart Fail. 2000 Sep;2(3):305-13. doi: 10.1016/s1388-9842(00)00094-5.

34. Engelmeier RS, Le TT, Kamalay S, Utecht KN, Nikstad TP, Kaliebe JW, et al. Randomized trial of high dose furosemide-hypertonic saline in acute decompensated heart failure with advanced renal disease. $J$ Am Coll Cardiol 2012; 59: E958. doi: 10.1016/S0735-1097(12)60959-0.

35. Licata G, Di Pasquale P, Parrinello G, Cardinale A, Scandurra $A$, Follone $G$, Argano C, Tuttolomondo A, Paterna S. Effects of highdose furosemide and small-volume hypertonic saline solution infusion in comparison with a high dose of furosemide as bolus in refractory congestive heart failure: long-term effects. Am Heart J. 2003 Mar;145(3):459-66. doi: 10.1067/mhj.2003.166.

36. Di Pasquale P, Sarullo FM, Paterna S. Novel strategies: challenge loop diuretics and sodium management in heart failure--part II. Congest Heart Fail. 2007 May-Jun;13(3):170-6. doi: 10.1111/j.15275299.2007.06534.x.

37. Jao GT, Chiong JR. Hyponatremia in acute decompensated heart failure: mechanisms, prognosis, and treatment options. Clin Cardiol. 2010 Nov;33(11):666-71. doi: 10.1002/clc.20822.

38. Morisawa $D$, Hirotani $S$, Oboshi $M$, Sugahara $M$, Fukui $M$, Ando $T$, et al. Combination of hypertonic saline and low-dose furosemide is an effective treatment for refractory congestive heart failure with hyponatremia. J Cardiol Cases. 2014 Feb 11;9(5):179-182. doi: 10.1016/j.jccase.2013.12.014. 